\title{
Evolution of the structural and magnetic properties of $\mathrm{Sr}_{2-x} \mathrm{Ca}_{x} \mathrm{CrSbO}_{6}$
}

\section{Electronic Supporting Information}

Emily C. Hunter and Peter D. Battle*

Inorganic Chemistry Laboratory, University of Oxford, South Parks Road, Oxford, OX1 3QR, U. K. 
Table S1 - Structural parameters of $\mathrm{Sr}_{1.75} \mathrm{Ca}_{0.25} \mathrm{CrSbO}_{6}, \mathrm{Sr}_{1.25} \mathrm{Ca}_{0.75} \mathrm{CrSbO}_{6}$ and $\mathrm{Sr}_{0.75} \mathrm{Ca}_{1.25} \mathrm{CrSbO}_{6}$ at room temperature, as refined from powder $\mathrm{x}$-ray diffraction data collected on I11 (Space Group P21/n)

\begin{tabular}{|c|c|c|c|c|}
\hline & & $\mathrm{Sr}_{1.75} \mathrm{Ca}_{0.25} \mathrm{CrSbO}_{6}$ & $\mathrm{Sr}_{1.25} \mathrm{Ca}_{0.75} \mathrm{CrSbO}_{6}$ & $\mathrm{Sr}_{0.75} \mathrm{Ca}_{1.25} \mathrm{CrSbO}_{6}$ \\
\hline a/ $\AA$ & & $5.565817(6)$ & 5.52108(9) & 5.49252(13) \\
\hline$b / \AA$ & & $5.543283(7)$ & $5.52028(10)$ & 5.49971(13) \\
\hline$c / \AA$ & & 7.831500(9) & 7.81132(8) & 7.76498(17) \\
\hline$\beta /^{\circ}$ & & $90.0031(5)$ & 89.993(4) & 90.1360(19) \\
\hline $\mathrm{V} / \AA^{3}$ & & 241.6245(5) & 238.073(6) & 234.558(9) \\
\hline $\mathrm{Sr} / \mathrm{Ca}$ & $x$ & $0.0013(2)$ & $0.9987(7)$ & $0.9995(5)$ \\
\hline \multirow[t]{5}{*}{$4 e$} & $y$ & $-0.00652(9)$ & $0.01593(8)$ & $0.0264(1)$ \\
\hline & $z$ & $0.74997(6)$ & $0.2492(9)$ & $0.2504(6)$ \\
\hline & $U_{\text {iso }} / \AA^{2}$ & $0.00993(4)$ & $0.01127(6)$ & $0.01159(9)$ \\
\hline & Sr Occupancy & 0.88 & 0.635 & 0.385 \\
\hline & Ca Occupancy & 0.12 & 0.365 & 0.615 \\
\hline $\mathrm{Cr} / \mathrm{Sb} 1$ & $U_{\text {iso }} / \AA^{2}$ & $0.00278(2)$ & $0.00336(2)$ & $0.00376(3)$ \\
\hline \multirow[t]{2}{*}{$2 d(1 / 200)$} & Cr Occupancy & $0.987(1)$ & 0.953(1) & $0.955(1)$ \\
\hline & Sb Occupancy & $0.013(1)$ & $0.047(1)$ & $0.045(1)$ \\
\hline $\mathrm{Cr} / \mathrm{Sb} 2$ & $U_{\text {iso }} / \AA^{2}$ & $0.00278(2)$ & $0.00336(2)$ & $0.00376(3)$ \\
\hline \multirow[t]{2}{*}{$2 c(1 / 201 / 2)$} & Cr Occupancy & $0.023(1)$ & $0.067(1)$ & $0.065(1)$ \\
\hline & Sb Occupancy & 0.977 (1) & 0.933(1) & $0.935(1)$ \\
\hline $\mathrm{O} 1$ & $x$ & $0.5464(4)$ & $0.0389(12)$ & $0.075(1)$ \\
\hline \multirow[t]{3}{*}{$4 e$} & $y$ & $0.0014(6)$ & $0.4932(5)$ & $0.4875(6)$ \\
\hline & $z$ & $0.74994(34)$ & $0.2475(11)$ & $0.2514(9)$ \\
\hline & $U_{\text {iso }} / \AA^{2}$ & $0.00741(25)$ & $0.0071(5)$ & $0.0077(4)$ \\
\hline $\mathrm{O} 2$ & $x$ & $0.7393(6)$ & $0.7201(23)$ & $0.7290(13)$ \\
\hline \multirow[t]{3}{*}{$4 e$} & $y$ & $0.2642(6)$ & $0.2831(21)$ & $0.2856(15)$ \\
\hline & $z$ & $0.0236(10)$ & $0.0355(19)$ & $0.0261(11)$ \\
\hline & $U_{\text {iso }} / \AA^{2}$ & $0.00741(25)$ & $0.0071(5)$ & $0.0077(4)$ \\
\hline O3 & $x$ & $0.7643(5)$ & $0.2274(20)$ & $0.2091(15)$ \\
\hline \multirow[t]{3}{*}{$4 e$} & $y$ & $0.7631(6)$ & $0.2332(22)$ & $0.2113(14)$ \\
\hline & $z$ & $0.0210(10)$ & $0.9628(22)$ & $0.9648(13)$ \\
\hline & $U_{\text {iso }} / \AA^{2}$ & $0.00741(25)$ & $0.0071(5)$ & $0.0077(4)$ \\
\hline O (all) occupancy & & 1.00 & 0.9950 & 0.9950 \\
\hline
\end{tabular}


Table S2 - Structural parameters of $\mathrm{Sr}_{0.50} \mathrm{Ca}_{1.50} \mathrm{CrSbO}_{6}, \mathrm{Sr}_{0.25} \mathrm{Ca}_{1.75} \mathrm{CrSbO}_{6}$ and $\mathrm{Ca}_{2} \mathrm{CrSbO}_{6}$ at room temperature, as refined from powder x-ray diffraction data collected on I11 (Space Group P21/n)

\begin{tabular}{|c|c|c|c|c|}
\hline & & $\mathrm{Sr}_{0.50} \mathrm{Ca}_{1.50} \mathrm{CrSbO}_{6}$ & $\mathrm{Sr}_{0.25} \mathrm{Ca}_{1.75} \mathrm{CrSbO}_{6}$ & $\mathrm{Ca}_{2} \mathrm{CrSbO}_{6}$ \\
\hline a/ $\AA$ & & $5.463425(33)$ & $5.437808(30)$ & $5.407974(12)$ \\
\hline$b / \AA$ & & $5.496490(32)$ & 5.492989(30) & $5.494512(11)$ \\
\hline$c / \AA$ & & 7.74688(5) & 7.72160(5) & $7.691100(17)$ \\
\hline$\beta /^{\circ}$ & & $90.0447(15)$ & $90.0712(12)$ & $90.11100(20)$ \\
\hline $\mathrm{V} / \AA^{3}$ & & 232.6361(24) & 230.6427(22) & 228.5343(8) \\
\hline $\mathrm{Sr} / \mathrm{Ca}$ & $x$ & $0.98978(29)$ & $0.99273(32)$ & $0.99167(16)$ \\
\hline \multirow[t]{5}{*}{$4 e$} & $y$ & $0.03094(8)$ & $0.03653(7)$ & $0.04340(7)$ \\
\hline & $z$ & $0.25095(14)$ & $0.25045(12)$ & $0.25023(9)$ \\
\hline & $U_{\text {iso }} / \AA^{2}$ & $0.0092(1)$ & $0.00876(9)$ & $0.00668(7)$ \\
\hline & Sr Occupancy & 0.255 & 0.125 & 0 \\
\hline & Ca Occupancy & 0.745 & 0.875 & 1 \\
\hline $\mathrm{Cr} / \mathrm{Sb} 1$ & $U_{\text {iso }} / \AA^{2}$ & $0.00337(2)$ & $0.00350(2)$ & $0.00335(2)$ \\
\hline \multirow[t]{2}{*}{$2 d(1 / 200)$} & Cr Occupancy & $0.941(1)$ & $0.954(1)$ & $0.952(1)$ \\
\hline & Sb Occupancy & $0.059(1)$ & $0.046(1)$ & $0.048(1)$ \\
\hline $\mathrm{Cr} / \mathrm{Sb} 2$ & $U_{\text {iso }} / \AA^{2}$ & $0.00337(2)$ & $0.00350(2)$ & $0.00335(2)$ \\
\hline \multirow[t]{2}{*}{$2 c(1 / 201 / 2)$} & Cr Occupancy & $0.079(1)$ & $0.056(1)$ & $0.058(1)$ \\
\hline & Sb Occupancy & $0.921(1)$ & $0.944(1)$ & $0.942(1)$ \\
\hline $\mathrm{O} 1$ & $x$ & $0.0641(6)$ & $0.0857(5)$ & $0.08743(30)$ \\
\hline \multirow[t]{3}{*}{$4 e$} & $y$ & $0.4830(4)$ & $0.4806(4)$ & $0.47652(30)$ \\
\hline & $z$ & $0.2444(8)$ & $0.2507(5)$ & $0.25043(25)$ \\
\hline & $U_{\text {iso }} / \AA^{2}$ & $0.00636(26)$ & $0.00656(22)$ & $0.0072(4)$ \\
\hline $\mathrm{O} 2$ & $x$ & $0.7067(7)$ & $0.7065(5)$ & $0.70787(33)$ \\
\hline \multirow[t]{3}{*}{$4 e$} & $y$ & $0.2937(6)$ & $0.2938(5)$ & $0.29601(32)$ \\
\hline & $z$ & $0.0408(9)$ & $0.0384(7)$ & $0.04261(31)$ \\
\hline & $U_{\text {iso }} / \AA^{2}$ & $0.00636(26)$ & $0.00656(22)$ & $0.0068(4)$ \\
\hline $\mathrm{O} 3$ & $x$ & $0.2178(6)$ & $0.2116(5)$ & $0.20344(33)$ \\
\hline \multirow[t]{3}{*}{$4 e$} & $y$ & $0.2180(6)$ & $0.2116(5)$ & $0.21001(33)$ \\
\hline & $z$ & $0.9586(9)$ & $0.9591(7)$ & $0.95676(32)$ \\
\hline & $U_{\text {iso }} / \AA^{2}$ & $0.00636(26)$ & $0.00656(22)$ & $0.0084(5)$ \\
\hline O (all) occupancy & & 0.995 & 1.00 & 1.00 \\
\hline
\end{tabular}


Table S3 - Bond lengths $(\AA)$ in $\mathrm{Sr}_{1.75} \mathrm{Ca}_{0.25} \mathrm{CrSbO}_{6}, \mathrm{Sr}_{1.25} \mathrm{Ca}_{0.75} \mathrm{CrSbO}_{6}$ and $\mathrm{Sr}_{0.75} \mathrm{Ca}_{1.25} \mathrm{CrSbO}_{6}$ at room temperature.

\begin{tabular}{|c|c|c|c|}
\hline & $\mathrm{Sr}_{1.75} \mathrm{Ca}_{0.25} \mathrm{CrSbO}_{6}$ & $\mathrm{Sr}_{1.25} \mathrm{Ca}_{0.75} \mathrm{CrSbO}_{6}$ & $\mathrm{Sr}_{0.75} \mathrm{Ca}_{1.25} \mathrm{CrSbO}_{6}$ \\
\hline $\mathrm{Sr} / \mathrm{Ca}-\mathrm{O} 1$ & $2.532(3)$ & $2.556(7)$ & $2.347(7)$ \\
\hline $\mathrm{Sr} / \mathrm{Ca}-\mathrm{O} 1$ & 2.741(3) & $2.644(3)$ & $2.570(3)$ \\
\hline $\mathrm{Sr} / \mathrm{Ca}-\mathrm{O} 1$ & 2.828(3) & 2.894(3) & 2.993(3) \\
\hline $\mathrm{Sr} / \mathrm{Ca}-\mathrm{O} 1$ & $3.035(3)$ & 2.971(7) & $3.162(7)$ \\
\hline $\mathrm{Sr} / \mathrm{Ca}-\mathrm{O} 2$ & $2.589(6)$ & 2.437(16) & $2.520(8)$ \\
\hline $\mathrm{Sr} / \mathrm{Ca}-\mathrm{O} 2$ & $2.696(6)$ & 2.707(16) & $2.689(10)$ \\
\hline $\mathrm{Sr} / \mathrm{Ca}-\mathrm{O} 2$ & 2.828(6) & $2.779(16)$ & $2.694(10)$ \\
\hline $\mathrm{Sr} / \mathrm{Ca}-\mathrm{O} 2$ & 2.995(6) & $3.175(16)$ & 3.130(8) \\
\hline $\mathrm{Sr} / \mathrm{Ca}-\mathrm{O} 3$ & $2.596(6)$ & 2.489(17) & $2.409(7)$ \\
\hline $\mathrm{Sr} / \mathrm{Ca}-\mathrm{O} 3$ & $2.717(6)$ & $2.635(16)$ & $2.700(13)$ \\
\hline $\mathrm{Sr} / \mathrm{Ca}-\mathrm{O} 3$ & $2.807(6)$ & 2.836(16) & $2.722(13)$ \\
\hline $\mathrm{Sr} / \mathrm{Ca}-\mathrm{O} 3$ & 2.987(6) & 3.128(18) & 3.231(7) \\
\hline$<\mathrm{Sr} / \mathrm{Ca}-\mathrm{O}>$ & 2.779 & 2.771 & 2.764 \\
\hline $\mathrm{Cr} / \mathrm{Sb} 1-\mathrm{O} 1$ & $1.975(3)$ & $1.984(9)$ & $1.976(7)$ \\
\hline $\mathrm{Cr} / \mathrm{Sb} 1-\mathrm{O} 2$ & $1.988(3)$ & $1.999(7)$ & $2.022(7)$ \\
\hline $\mathrm{Cr} / \mathrm{Sb} 1-\mathrm{O} 3$ & $1.979(3)$ & $2.002(7)$ & 1.994(6) \\
\hline$<\mathrm{Cr} / \mathrm{Sb} 1-\mathrm{O}>$ & 1.981 & 1.995 & 1.997 \\
\hline $\mathrm{Cr} / \mathrm{Sb} 2-\mathrm{O} 1$ & $1.974(3)$ & $1.946(9)$ & $1.995(7)$ \\
\hline $\mathrm{Cr} / \mathrm{Sb} 2-\mathrm{O} 2$ & $1.961(3)$ & $1.957(8)$ & $1.910(7)$ \\
\hline $\mathrm{Cr} / \mathrm{Sb} 2-\mathrm{O} 3$ & $1.969(3)$ & $1.974(8)$ & $1.979(6)$ \\
\hline$<\mathrm{Cr} / \mathrm{Sb} 2-\mathrm{O}>$ & 1.968 & 1.959 & 1.961 \\
\hline
\end{tabular}


Table S4 - Bond lengths $(\AA)$ in $\mathrm{Sr}_{0.50} \mathrm{Ca}_{1.50} \mathrm{CrSbO}_{6}, \mathrm{Sr}_{0.25} \mathrm{Ca}_{1.75} \mathrm{CrSbO}_{6}$ and $\mathrm{Ca}_{2} \mathrm{CrSbO}_{6}$ at room temperature.

\begin{tabular}{|c|c|c|c|}
\hline & $\mathrm{Sr}_{0.50} \mathrm{Ca}_{1.50} \mathrm{CrSbO}_{6}$ & $\mathrm{Sr}_{0.25} \mathrm{Ca}_{1.75} \mathrm{CrSbO}_{6}$ & $\mathrm{Ca}_{2} \mathrm{CrSbO}_{6}$ \\
\hline $\mathrm{Sr} / \mathrm{Ca}-\mathrm{O} 1$ & $2.452(4)$ & $2.313(3)$ & $2.3057(18)$ \\
\hline $\mathrm{Sr} / \mathrm{Ca}-\mathrm{O} 1$ & $2.518(2)$ & $2.491(2)$ & 2.4355(17) \\
\hline $\mathrm{Sr} / \mathrm{Ca}-\mathrm{O} 1$ & $3.038(4)$ & $3.095(2)$ & 3.1532(18) \\
\hline $\mathrm{Sr} / \mathrm{Ca}-\mathrm{O} 1$ & $3.039(2)$ & $3.160(3)$ & $3.1575(17)$ \\
\hline $\mathrm{Sr} / \mathrm{Ca}-\mathrm{O} 2$ & $2.336(6)$ & $2.370(4)$ & $2.357(2)$ \\
\hline $\mathrm{Sr} / \mathrm{Ca}-\mathrm{O} 2$ & $2.669(5)$ & $2.663(4)$ & $2.612(2)$ \\
\hline $\mathrm{Sr} / \mathrm{Ca}-\mathrm{O} 2$ & $2.715(6)$ & $2.675(5)$ & $2.682(2)$ \\
\hline $\mathrm{Sr} / \mathrm{Ca}-\mathrm{O} 2$ & $3.324(6)$ & $3.309(4)$ & $3.347(2)$ \\
\hline $\mathrm{Sr} / \mathrm{Ca}-\mathrm{O} 3$ & $2.406(5)$ & $2.389(4)$ & $2.362(2)$ \\
\hline $\mathrm{Sr} / \mathrm{Ca}-\mathrm{O} 3$ & $2.589(5)$ & $2.618(4)$ & $2.607(2)$ \\
\hline $\mathrm{Sr} / \mathrm{Ca}-\mathrm{O} 3$ & $2.783(6)$ & $2.722(5)$ & $2.693(2)$ \\
\hline $\mathrm{Sr} / \mathrm{Ca}-\mathrm{O} 3$ & $3.251(5)$ & $3.285(4)$ & 3.337(2) \\
\hline$<\mathrm{Sr} / \mathrm{Ca}-\mathrm{O}>$ & 2.760 & 2.758 & 2.754 \\
\hline $\mathrm{Cr} / \mathrm{Sb} 1-\mathrm{O} 1$ & $2.013(6)$ & $1.984(4)$ & $1.9819(19)$ \\
\hline $\mathrm{Cr} / \mathrm{Sb} 1-\mathrm{O} 2$ & $1.995(4)$ & 1.988(3) & 2.0037 (18) \\
\hline $\mathrm{Cr} / \mathrm{Sb} 1-\mathrm{O} 3$ & $1.979(4)$ & $1.977(3)$ & $2.0030(18)$ \\
\hline$<\mathrm{Cr} / \mathrm{Sb} 1-\mathrm{O}>$ & 1.996 & 1.983 & 1.996 \\
\hline $\mathrm{Cr} / \mathrm{Sb} 2-\mathrm{O} 1$ & $1.927(6)$ & $1.993(4)$ & $1.987(2)$ \\
\hline $\mathrm{Cr} / \mathrm{Sb} 2-\mathrm{O} 2$ & $1.988(4)$ & $1.980(3)$ & $1.9651(18)$ \\
\hline $\mathrm{Cr} / \mathrm{Sb} 2-\mathrm{O} 3$ & $1.981(4)$ & $1.983(3)$ & $1.9650(18)$ \\
\hline$<\mathrm{Cr} / \mathrm{Sb} 2-\mathrm{O}>$ & 1.965 & 1.985 & 1.972 \\
\hline
\end{tabular}


Table S5 - Key bond angles $\left({ }^{\circ}\right)$ in $\mathrm{Sr}_{1.75} \mathrm{Ca}_{0.25} \mathrm{CrSbO}_{6}, \mathrm{Sr}_{1.25} \mathrm{Ca}_{0.75} \mathrm{CrSbO}_{6}$ and $\mathrm{Sr}_{1.25} \mathrm{Ca}_{0.75} \mathrm{CrSbO}_{6}$ at room temperature.

\begin{tabular}{|llll|}
\hline & $\mathrm{Sr}_{1.75} \mathrm{Ca}_{0.25} \mathrm{CrSbO}_{6}$ & $\mathrm{Sr}_{1.25} \mathrm{Ca}_{0.75} \mathrm{CrSbO}_{6}$ & $\mathrm{Sr}_{0.75} \mathrm{Ca}_{1.25} \mathrm{CrSbO}_{6}$ \\
\hline $\mathrm{O} 1-\mathrm{Cr} / \mathrm{Sb} 1-\mathrm{O} 2$ & $90.09(25)$ & $93.3(4)$ & $93.44(28)$ \\
$\mathrm{O} 1-\mathrm{Cr} / \mathrm{Sb} 1-\mathrm{O} 3$ & $90.70(25)$ & $94.3(6)$ & $90.8(4)$ \\
$\mathrm{O} 2-\mathrm{Cr} / \mathrm{Sb} 1-\mathrm{O} 3$ & $90.97(11)$ & $91.5(9)$ & $93.4(5)$ \\
$<\mathrm{O}-\mathrm{Cr} / \mathrm{Sb} 1-\mathrm{O}>$ & 90.59 & 93.03 & 92.55 \\
& & & \\
$\mathrm{O} 1-\mathrm{Cr} / \mathrm{Sb} 2-\mathrm{O} 2$ & $90.05(25)$ & $93.7(4)$ & $91.95(29)$ \\
$\mathrm{O} 1-\mathrm{Cr} / \mathrm{Sb} 2-\mathrm{O} 3$ & $90.41(25)$ & $93.6(6)$ & $90.63(33)$ \\
$\mathrm{O} 2-\mathrm{Cr} / \mathrm{Sb} 2-\mathrm{O} 3$ & $90.41(11)$ & $93.8(9)$ & $91.6(5)$ \\
$<\mathrm{O}-\mathrm{Cr} / \mathrm{Sb} 2-\mathrm{O}>$ & 90.29 & 93.7 & 91.39 \\
& & & $155.72(35)$ \\
$\mathrm{Cr} / \mathrm{Sb} 1-\mathrm{O} 1-\mathrm{Cr} / \mathrm{Sb} 2$ & $164.95(14)$ & $167.2(4)$ & $162.5(4)$ \\
$\mathrm{Cr} / \mathrm{Sb} 1-\mathrm{O} 2-\mathrm{Cr} / \mathrm{Sb} 2$ & $167.8(4)$ & $158.5(8)$ & $156.03(34)$ \\
$\mathrm{Cr} / \mathrm{Sb} 1-\mathrm{O} 3-\mathrm{Cr} / \mathrm{Sb} 2$ & $168.6(4)$ & $160.9(10)$ & 158.08 \\
$<\mathrm{Cr} / \mathrm{Sb} 1-\mathrm{O}-\mathrm{Cr} / \mathrm{Sb} 2>$ & 167.12 & 162.2 & \\
\hline
\end{tabular}

Table S6 - Key bond angles $\left({ }^{\circ}\right)$ in $\mathrm{Sr}_{0.50} \mathrm{Ca}_{1.50} \mathrm{CrSbO}_{6}, \mathrm{Sr}_{0.25} \mathrm{Ca}_{1.75} \mathrm{CrSbO}_{6}$ and $\mathrm{Ca}_{2} \mathrm{CrSbO}_{6}$ at room temperature.

\begin{tabular}{|llll|}
\hline & $\mathrm{Sr}_{0.50} \mathrm{Ca}_{1.50} \mathrm{CrSbO}_{6}$ & $\mathrm{Sr}_{0.25} \mathrm{Ca}_{1.75} \mathrm{CrSbO}_{6}$ & $\mathrm{Ca}_{2} \mathrm{CrSbO}_{6}$ \\
\hline $\mathrm{O} 1-\mathrm{Cr} / \mathrm{Sb} 1-\mathrm{O} 2$ & $91.12(23)$ & $91.85(18)$ & $91.68(8)$ \\
$\mathrm{O} 1-\mathrm{Cr} / \mathrm{Sb} 1-\mathrm{O} 3$ & $92.95(24)$ & $90.02(17)$ & $90.33(9)$ \\
$\mathrm{O} 2-\mathrm{Cr} / \mathrm{Sb} 1-\mathrm{O} 3$ & $91.33(14)$ & $90.32(12)$ & $90.48(8)$ \\
$<\mathrm{O}-\mathrm{Cr} / \mathrm{Sb} 1-\mathrm{O}>$ & 91.80 & 90.73 & 90.83 \\
& & & \\
$\mathrm{O} 1-\mathrm{Cr} / \mathrm{Sb} 2-\mathrm{O} 2$ & $92.18(21)$ & $90.65(17)$ & $90.51(9)$ \\
$\mathrm{O} 1-\mathrm{Cr} / \mathrm{Sb} 2-\mathrm{O} 3$ & $90.72(25)$ & $91.31(18)$ & $91.20(9)$ \\
$\mathrm{O} 2-\mathrm{Cr} / \mathrm{Sb} 2-\mathrm{O} 3$ & $93.65(15)$ & $91.99(12)$ & $90.93(8)$ \\
$<\mathrm{O}-\mathrm{Cr} / \mathrm{Sb} 2-\mathrm{O}>$ & 92.18 & 91.32 & 90.88 \\
& & & $151.40(9)$ \\
$\mathrm{Cr} / \mathrm{Sb} 1-\mathrm{O} 1-\mathrm{Cr} / \mathrm{Sb} 2$ & $158.79(19)$ & $152.19(14)$ & $152.46(12)$ \\
$\mathrm{Cr} / \mathrm{Sb} 1-\mathrm{O} 2-\mathrm{Cr} / \mathrm{Sb} 2$ & $153.16(31)$ & $153.83(24)$ & $152.55(12)$ \\
$\mathrm{Cr} / \mathrm{Sb} 1-\mathrm{O} 3-\mathrm{Cr} / \mathrm{Sb} 2$ & $156.32(34)$ & $154.72(24)$ & 152.14 \\
$<\mathrm{Cr} / \mathrm{Sb} 1-\mathrm{O}-\mathrm{Cr} / \mathrm{Sb} 2>$ & 156.09 & 153.58 & \\
\hline
\end{tabular}


Table S7 - Compositional analysis of the phases present in each sample

\begin{tabular}{|l|l|l|l|}
\hline $\begin{array}{l}\text { Nominal } \\
\text { composition }\end{array}$ & $\begin{array}{l}\text { Impurity } \\
\text { composition }\end{array}$ & $\begin{array}{l}\text { Impurity } \\
\text { percentage } \\
\text { (mol \%) }\end{array}$ & $\begin{array}{l}\text { Adjusted } \\
\text { composition }\end{array}$ \\
\hline $\mathrm{Sr}_{2} \mathrm{CrSbO}_{6}$ & $\mathrm{n} / \mathrm{a}$ & 0 & $\mathrm{Sr}_{2} \mathrm{CrSbO}_{6}$ \\
$\mathrm{Sr}_{1.75} \mathrm{Ca}_{0.25} \mathrm{CrSbO}_{6}$ & $\mathrm{Sr}_{0.84} \mathrm{Ca}_{1.16} \mathrm{Sb}_{2} \mathrm{O}_{7}$ & 0.91 & $\mathrm{Sr}_{1.76} \mathrm{Ca}_{0.24} \mathrm{Cr}_{1.01} \mathrm{Sb}_{0.99} \mathrm{O}_{6}$ \\
$\mathrm{Sr}_{1.5} \mathrm{Ca}_{0.5} \mathrm{CrSbO}_{6}$ & $\mathrm{Sr}_{0.61} \mathrm{Ca}_{1.39} \mathrm{Sb}_{2} \mathrm{O}_{7}$ & 1.81 & $\mathrm{Sr}_{1.55} \mathrm{Ca}_{0.45} \mathrm{Cr}_{1.02} \mathrm{Sb}_{0.98} \mathrm{O}_{6} \quad$ and \\
$\mathrm{Sr}_{1.25} \mathrm{Ca}_{0.75} \mathrm{CrSbO}_{6}$ & $\mathrm{Sr}_{0.41} \mathrm{Ca}_{1.59} \mathrm{Sb}_{2} \mathrm{O}_{7}$ & 2.59 & $\mathrm{Sr}_{1.33} \mathrm{Ca}_{0.67} \mathrm{Cr}_{1.02} \mathrm{Sb}_{0.98} \mathrm{O}_{6}$ \\
$\mathrm{SrCaCrSbO}_{6}$ & $\mathrm{Sr}_{0.29} \mathrm{Ca}_{1.71} \mathrm{Sb}_{2} \mathrm{O}_{7}$ & 4.16 & $\mathrm{Sr}_{1.27} \mathrm{Ca}_{0.73} \mathrm{Cr}_{1.02} \mathrm{Sb}_{0.98} \mathrm{O}_{5.97}$ \\
$\mathrm{Sr}_{0.75} \mathrm{Ca}_{1.25} \mathrm{CrSbO}_{6}$ & $\mathrm{Sr}_{0.28} \mathrm{Ca}_{1.72} \mathrm{Sb}_{2} \mathrm{O}_{7}$ & 2.56 & $\mathrm{Sr}_{1.03} \mathrm{Ca}_{0.97} \mathrm{Cr}_{1.04} \mathrm{Sb}_{0.95} \mathrm{O}_{5.94}$ \\
$\mathrm{Sr}_{0.5} \mathrm{Ca}_{1.5} \mathrm{CrSbO}_{6}$ & $\mathrm{Sr}_{0.26} \mathrm{Ca}_{1.74} \mathrm{Sb}_{2} \mathrm{O}_{7}$ & 2.33 & $\mathrm{Sr}_{0.51} \mathrm{Ca}_{1.49} \mathrm{Cr}_{1.02} \mathrm{Sb}_{0.98} \mathrm{Sb}_{0.98} \mathrm{O}_{5.97}$ \\
$\mathrm{Sr}_{0.25} \mathrm{Ca}_{1.75} \mathrm{CrSbO}_{6}$ & $\mathrm{Sr}_{0.19} \mathrm{Ca}_{1.81} \mathrm{Sb}_{2} \mathrm{O}_{7}$ & 1.16 & $\mathrm{Sr}_{0.25} \mathrm{Ca}_{1.75} \mathrm{Cr}_{1.01} \mathrm{Sb}_{0.99} \mathrm{O}_{6}$ \\
$\mathrm{Ca}_{2} \mathrm{CrSbO}_{6}$ & $\mathrm{Ca}_{2} \mathrm{Sb}_{2} \mathrm{O}_{7}$ & 1.02 & $\mathrm{Ca}_{2} \mathrm{Cr}_{1.01} \mathrm{Sb}_{0.99} \mathrm{O}_{6}$ \\
\hline
\end{tabular}




\section{Figure captions}

Figure S1 - Observed (red crosses) and calculated (green line) neutron powder diffraction profiles of $\mathrm{Sr}_{2} \mathrm{CrSbO}_{6}$ at $25 \mathrm{~K}$. Data were collected on D2b and fitted using the space group $I \overline{1}$. Reflection markers for $\mathrm{Sr}_{2} \mathrm{CrSbO}_{6}$ are shown in black.

Figure S2 - Observed (red crosses) and calculated (green line) neutron powder diffraction profiles of nominal $\mathrm{Sr}_{1.5} \mathrm{Ca}_{0.5} \mathrm{CrSbO}_{6}$ collected at room temperature on $\mathrm{D} 2 \mathrm{~b}$. The data were refined using a two-phase model where both perovskite phases are of the space group $P 2_{1} / n$. Reflection markers are shown top to bottom for $\mathrm{Sr}_{0.61} \mathrm{Ca}_{1.39} \mathrm{Sb}_{2} \mathrm{O}_{7}$ (blue), $P 2{ }_{1} / n$ $\mathrm{Sr}_{1.33} \mathrm{Ca}_{0.67} \mathrm{Cr}_{1.02} \mathrm{Sb}_{0.98} \mathrm{O}_{6}$ (red) and $P 2_{1} / n \mathrm{Sr}_{1.55} \mathrm{Ca}_{0.45} \mathrm{Cr}_{1.02} \mathrm{Sb}_{0.98} \mathrm{O}_{6}$ (black).

Figure S3 - Observed (red crosses) and calculated (green line) neutron powder diffraction profiles of nominal $\mathrm{Sr}_{1.5} \mathrm{Ca}_{0.5} \mathrm{CrSbO}_{6}$ collected at $25 \mathrm{~K}$ on D2b. The data were refined using a two-phase model where both perovskite phases are of the space group $P 21 / n$. Reflection markers are shown top to bottom for $\mathrm{Sr}_{0.61} \mathrm{Ca}_{1.39} \mathrm{Sb}_{2} \mathrm{O}_{7}$ (blue), $P 2{ }_{1} / n \mathrm{Sr}_{1.33} \mathrm{Ca}_{0.67} \mathrm{Cr}_{1.02} \mathrm{Sb}_{0.98} \mathrm{O}_{6}$ (red) and $P 2{ }_{1} / n \mathrm{Sr}_{1.55} \mathrm{Ca}_{0.45} \mathrm{Cr}_{1.02} \mathrm{Sb}_{0.98} \mathrm{O}_{6}$ (black).

Figure S4 - Observed (red crosses) and calculated (green line) neutron powder diffraction profiles of nominal $\mathrm{Sr}_{1.5} \mathrm{Ca}_{0.5} \mathrm{CrSbO}_{6}$ collected at $2 \mathrm{~K}$ on D2b. The data were refined using a two-phase model where both perovskite phases are of the space group $P 2_{1} / n$. Reflection markers are shown top to bottom for $\mathrm{Sr}_{0.61} \mathrm{Ca}_{1.39} \mathrm{Sb}_{2} \mathrm{O}_{7}$ (blue), $P 2{ }_{1} / n \mathrm{Sr}_{1.33} \mathrm{Ca}_{0.67} \mathrm{Cr}_{1.02} \mathrm{Sb}_{0.98} \mathrm{O}_{6}$ (red) and $P 2{ }_{1} / n \mathrm{Sr}_{1.55} \mathrm{Ca}_{0.45} \mathrm{Cr}_{1.02} \mathrm{Sb}_{0.98} \mathrm{O}_{6}$ (black).

Figure S5 - Observed (red crosses) and calculated (green line) neutron powder diffraction profiles of $\mathrm{SrCaCrSbO}_{6}$ at room temperature. Data were collected on D2b and fitted using the space group $P 2_{1} / n$. Reflection markers are shown top to bottom for $\mathrm{Sr}_{0.29} \mathrm{Ca}_{1.71} \mathrm{Sb}_{2} \mathrm{O}_{7}$ (red) and $\mathrm{SrCaCrSbO}_{6}$ (black).

Figure S6 - Observed (red crosses) and calculated (green line) neutron powder diffraction profiles of $\mathrm{SrCaCrSbO}_{6}$ at $25 \mathrm{~K}$. Data were collected on D2b and fitted using the space group $P 2_{1} / n$. Reflection markers are shown top to bottom for $\mathrm{Sr}_{0.29} \mathrm{Ca}_{1.71} \mathrm{Sb}_{2} \mathrm{O}_{7}$ (red) and $\mathrm{SrCaCrSbO}_{6}$ (black).

Figure S7 - Observed (red crosses) and calculated (green line) neutron powder diffraction profiles of $\mathrm{SrCaCrSbO}_{6}$ at $2 \mathrm{~K}$. Data were collected on $\mathrm{D} 2 \mathrm{~b}$ and fitted using the space group 
$P 2_{1} / n$. Reflection markers are shown top to bottom for $\mathrm{Sr}_{0.29} \mathrm{Ca}_{1.71} \mathrm{Sb}_{2} \mathrm{O}_{7}$ (blue), magnetic $\mathrm{SrCaCrSbO}_{6}$ (red) and structural $\mathrm{SrCaCrSbO}_{6}$ (black).

Figure S8 - Observed (red crosses) and calculated (green line) neutron powder diffraction profiles of $\mathrm{SrCaCrSbO}_{6}$ at $2 \mathrm{~K}$. Data were collected on $\mathrm{D} 1 \mathrm{~b}$ and fitted using the space group $P 2_{1} / n$. Reflection markers are shown top to bottom for magnetic $\mathrm{SrCaCrSbO}_{6}$ (blue), $\mathrm{Sr}_{0.29} \mathrm{Ca}_{1.71} \mathrm{Sb}_{2} \mathrm{O}_{7}$ (red) and structural $\mathrm{SrCaCrSbO}_{6}$ (black).

Figure S9 - Observed (red crosses) and calculated (green line) synchrotron x-ray diffraction profiles of a) $\mathrm{Sr}_{1.75} \mathrm{Ca}_{0.25} \mathrm{CrSbO}_{6}$ and b) $\mathrm{Ca}_{2} \mathrm{CrSbO}_{6}$ collected at room temperature on $\mathrm{I} 11$ and fitted using the space group $P 2_{1} / n$. Reflection markers are shown top to bottom for $\mathrm{Sr}_{1}$ ${ }_{x} \mathrm{Ca}_{x} \mathrm{Sb}_{2} \mathrm{O}_{7}$ (red) and structural $\mathrm{Sr}_{1-x} \mathrm{Ca}_{x} \mathrm{CrSbO}_{6}$ (black). Inset is a close-up of the XRD pattern between 10 and $22^{\circ}$. 


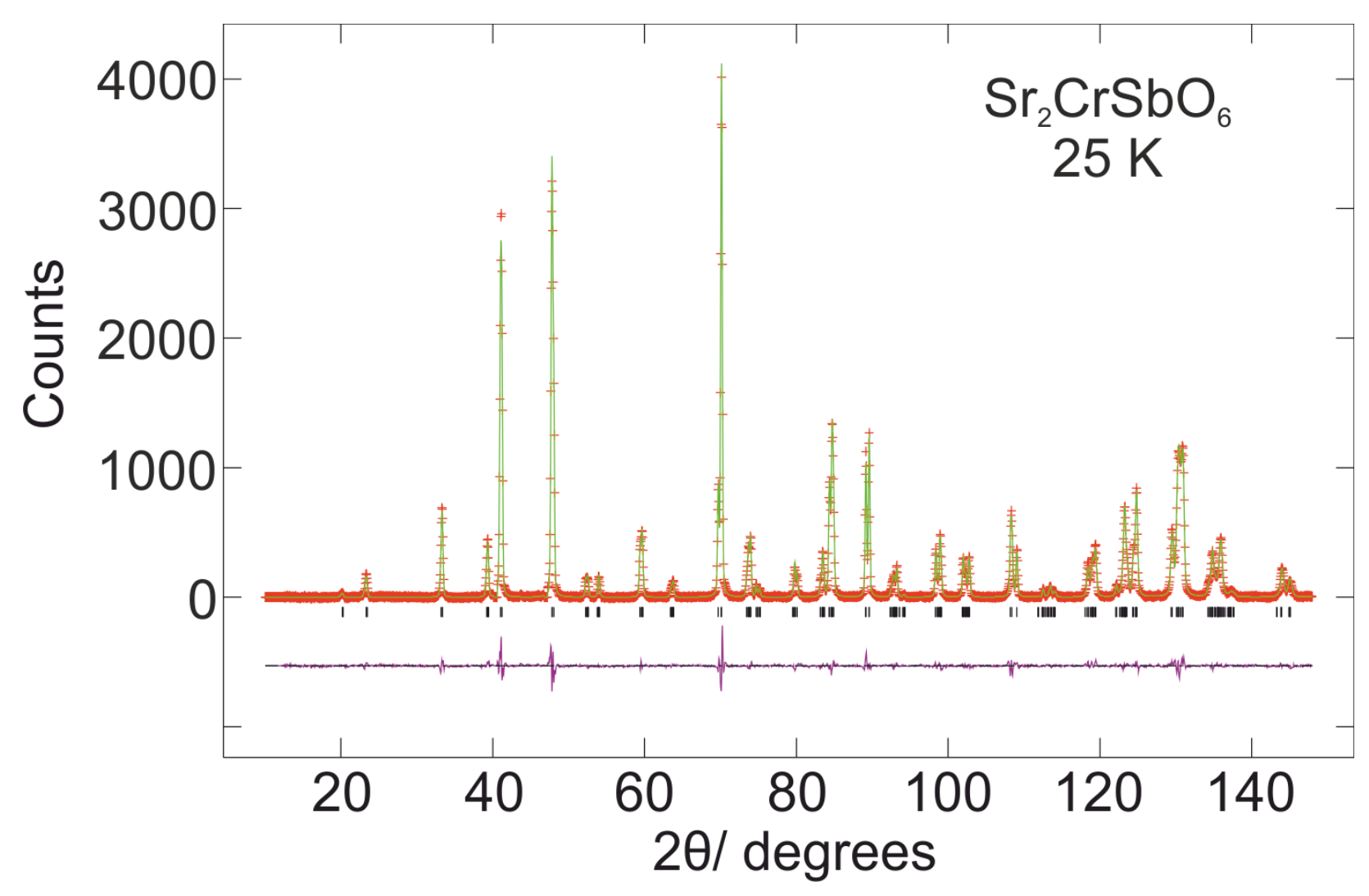

Figure S1

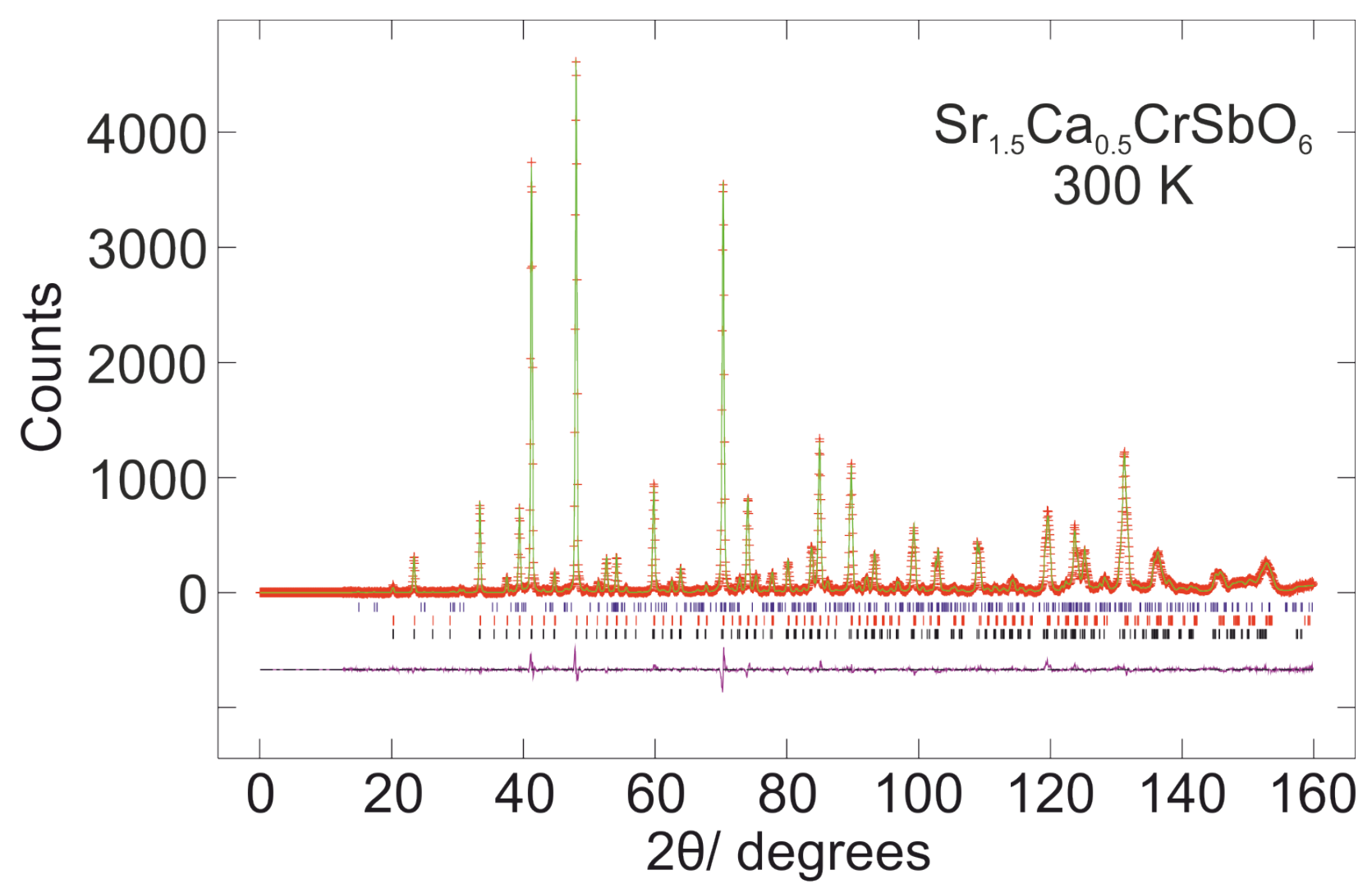

Figure S2 


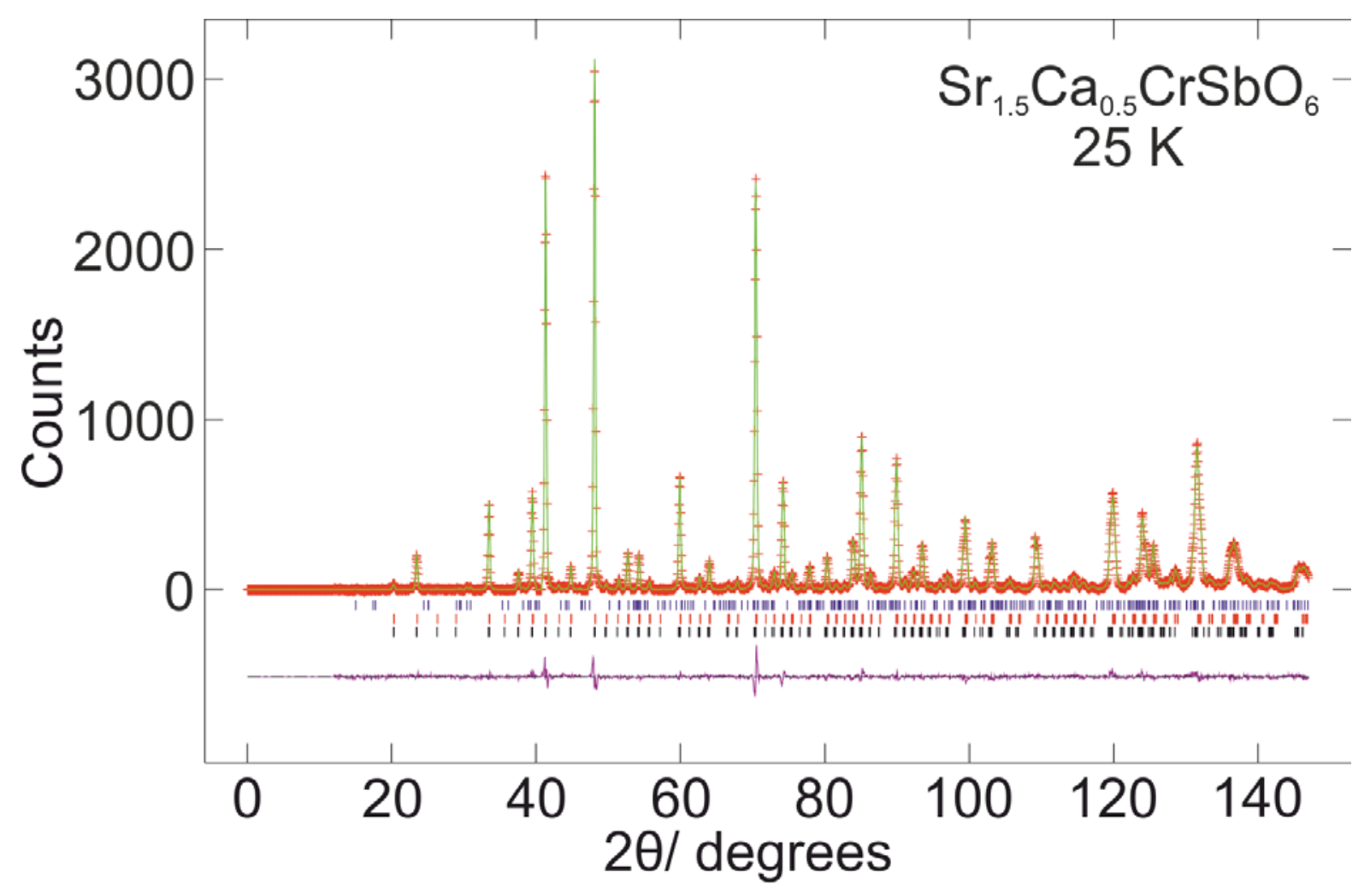

Figure S3

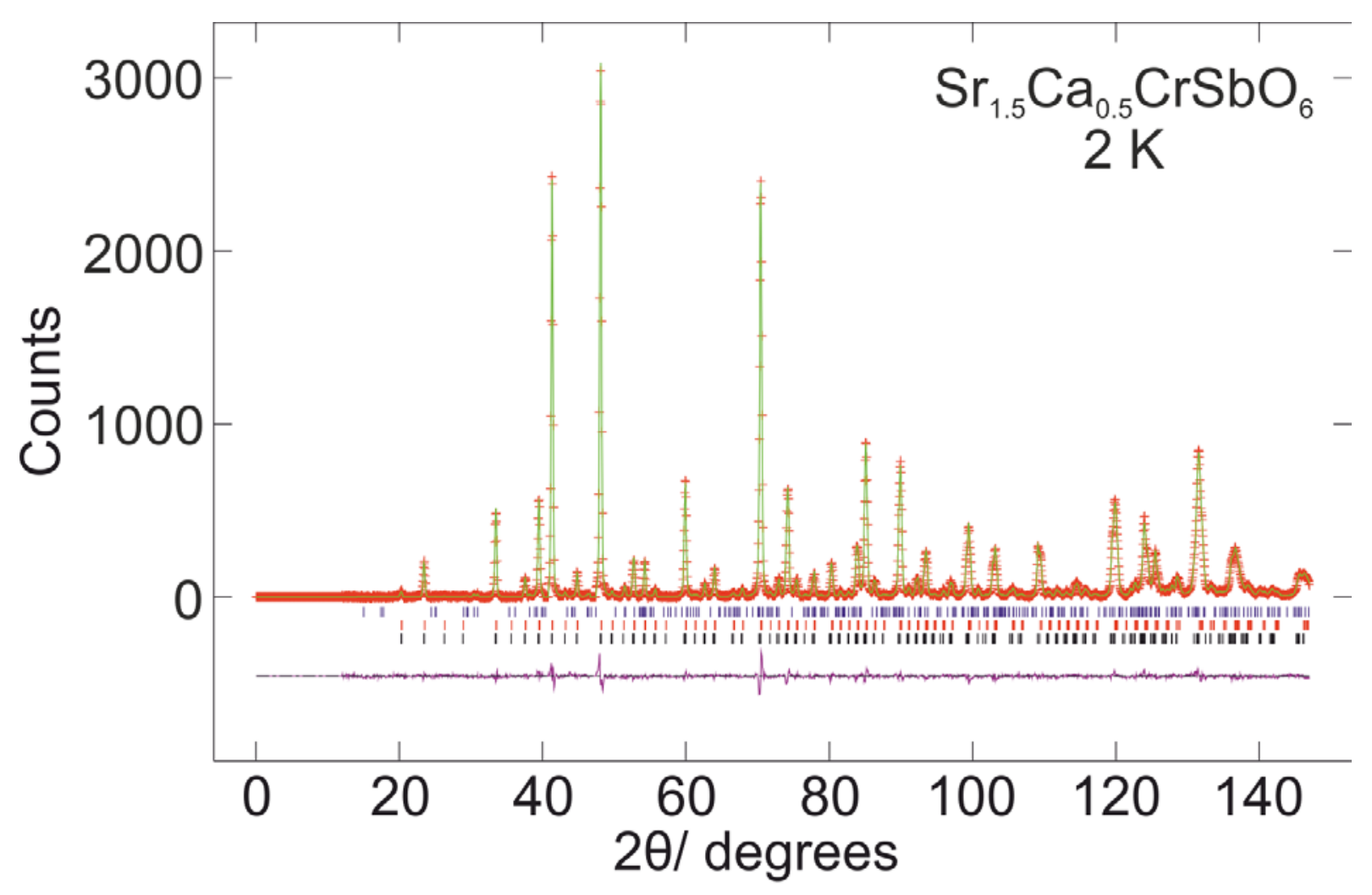

Figure S4 


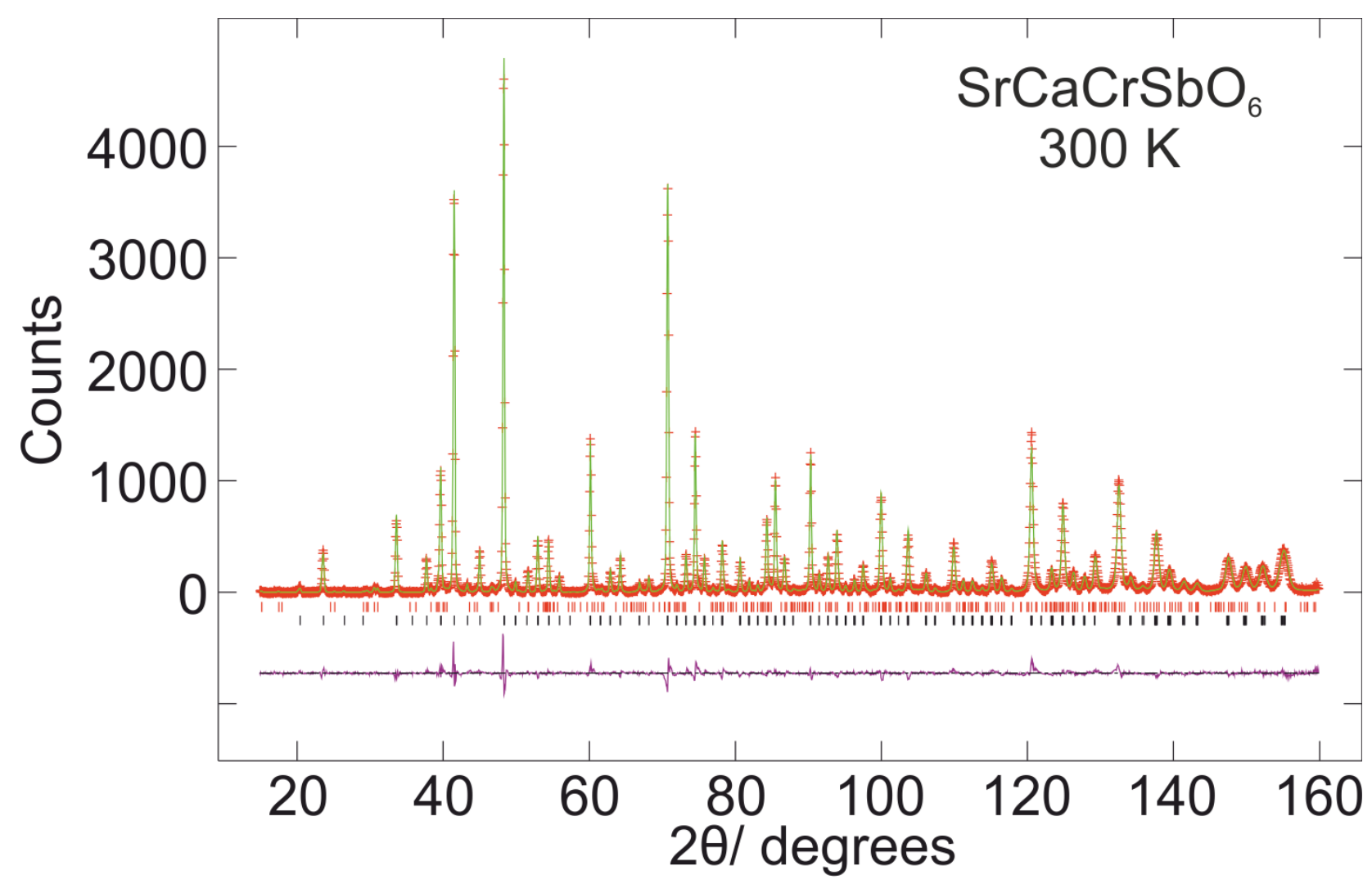

Figure S5

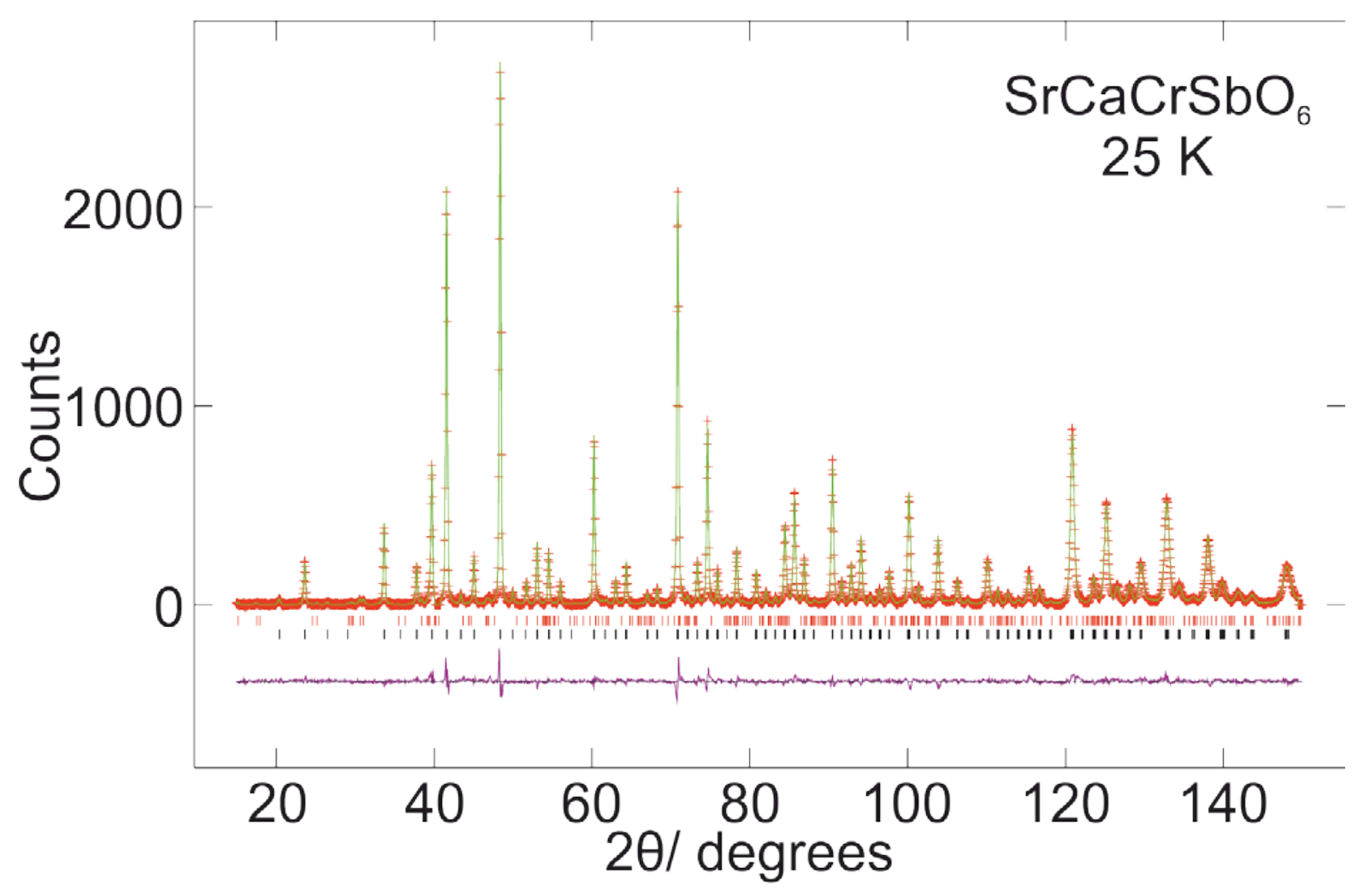

Figure S6 


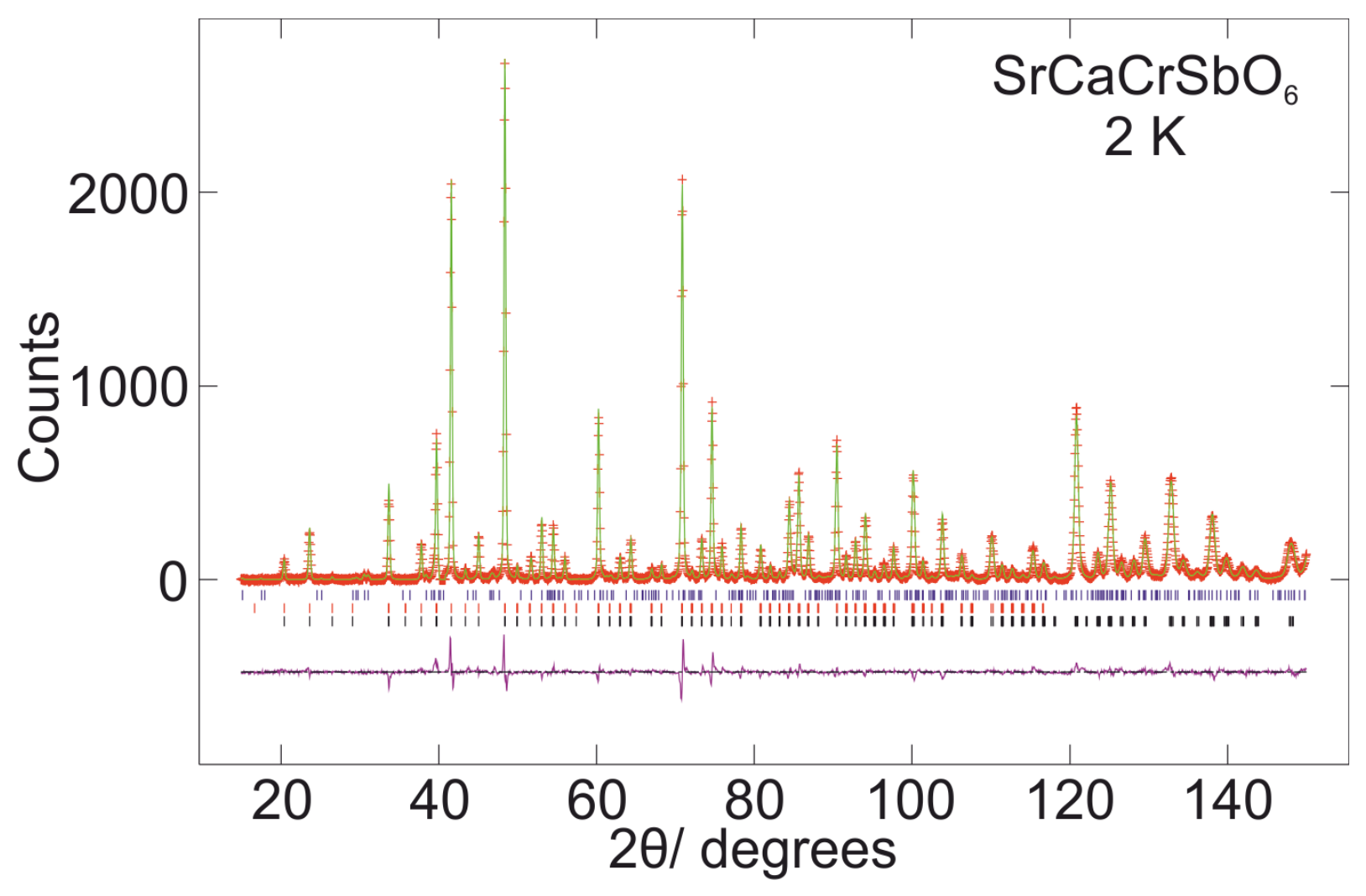

Figure S7

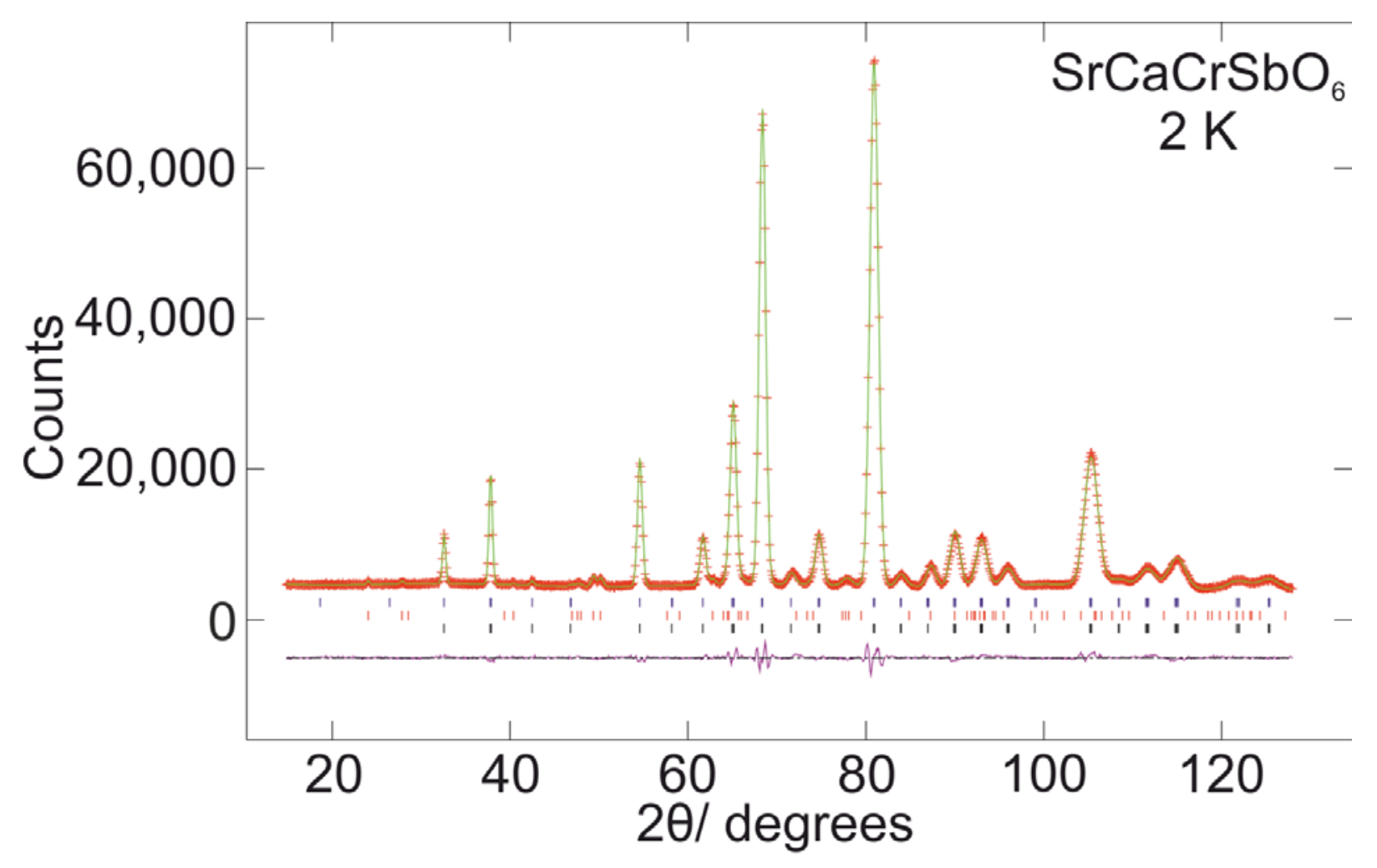

Figure S8 
a)

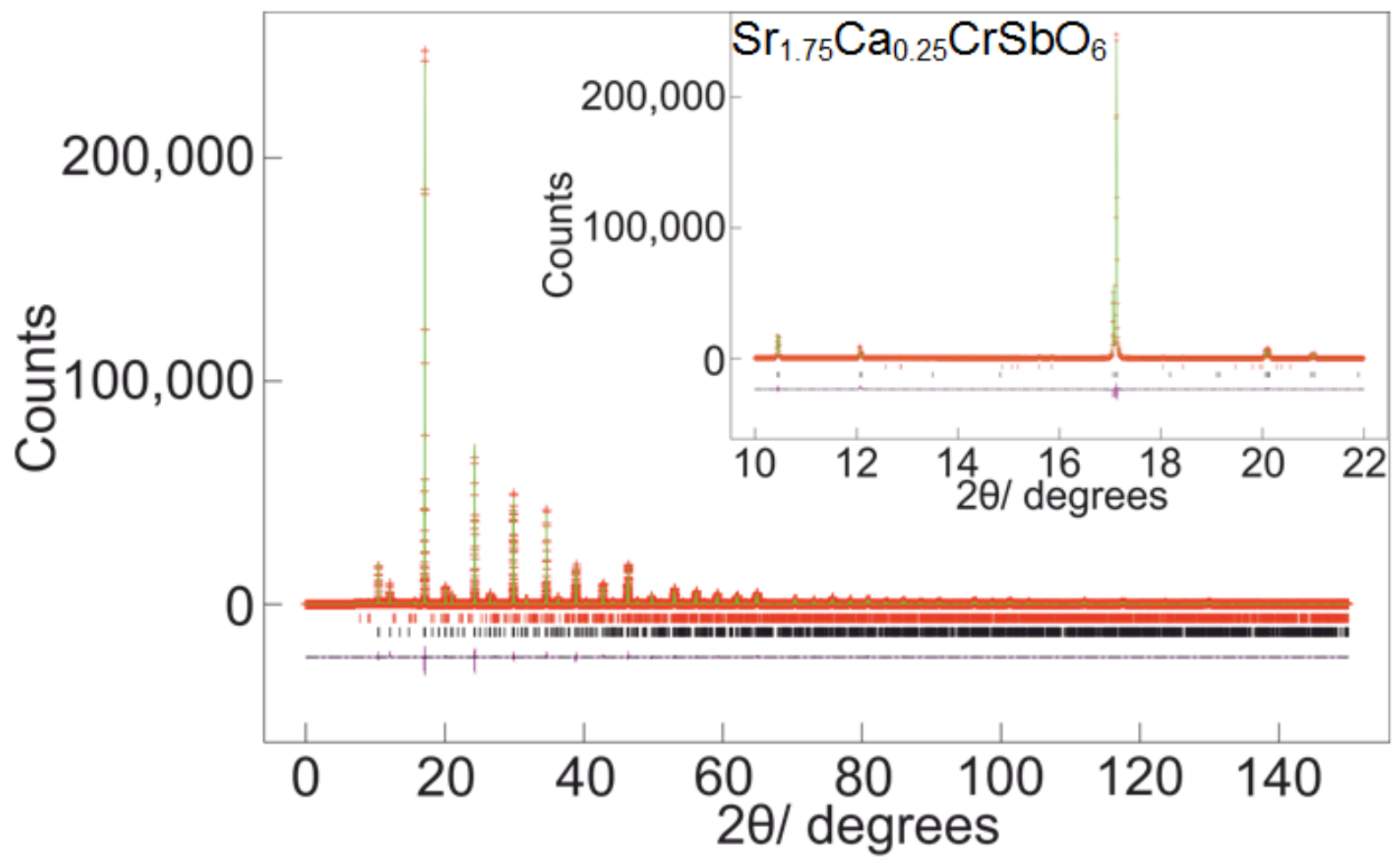

b)

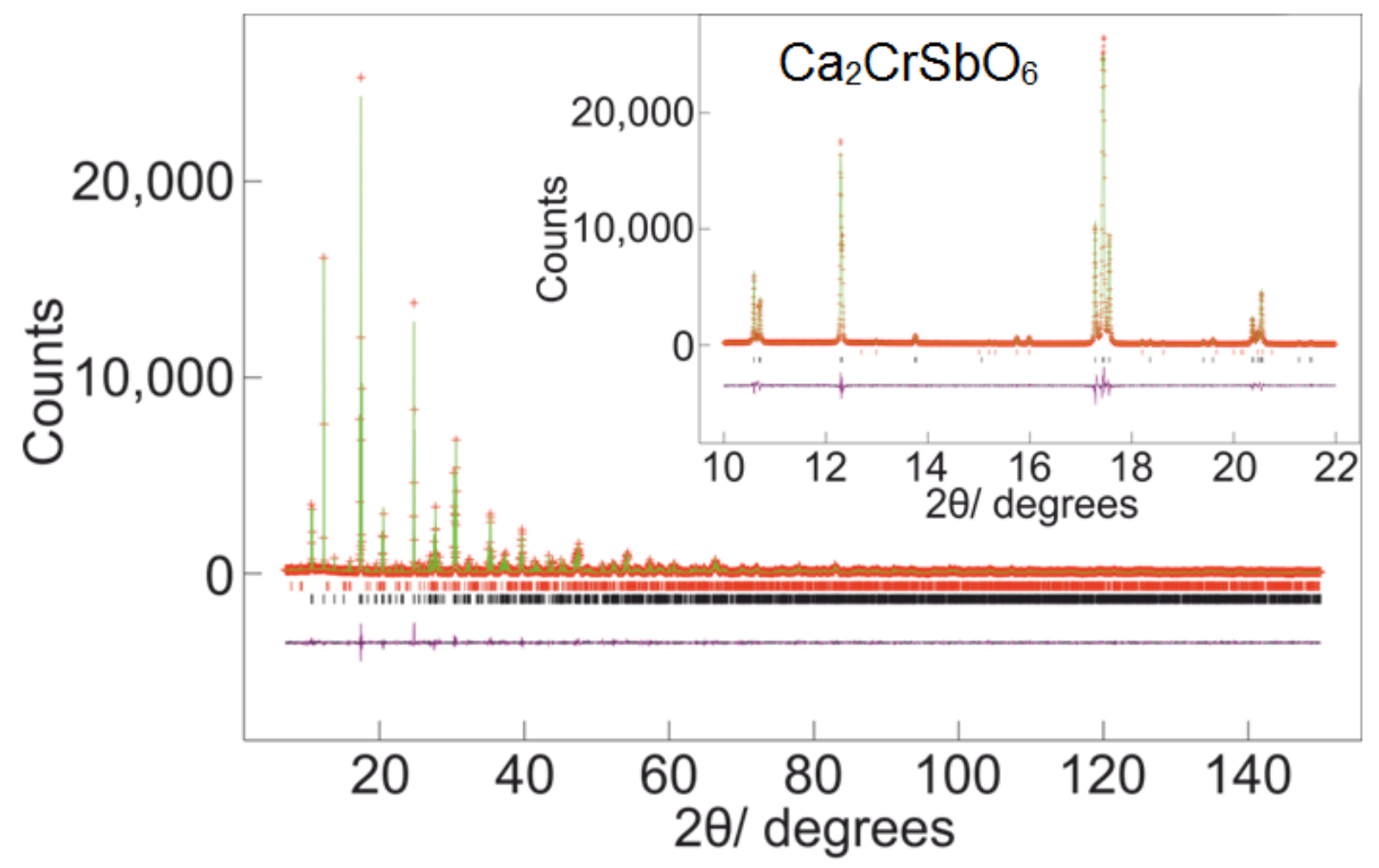

Figure S9 